\title{
Sound and feeling: Musical training moderates the association between adult ADHD and emotion regulation
}

\author{
Michael Tate ${ }^{1 \dagger}$ \\ David M. Greenberg, $\mathrm{PhD}^{2,3}$ \\ Sarah O’Neill, $\mathrm{PhD}^{1,4}$ \\ ${ }^{1}$ The City College of New York, City University of New York, New York City, NY, USA \\ ${ }^{2}$ Bar-Ilan University, Ramat Gan, Tel Aviv, Israel \\ ${ }^{3}$ University of Cambridge, Cambridge, United Kingdom \\ ${ }^{4}$ The Graduate Center, City University of New York, New York City, NY, USA \\ ${ }^{\dagger}$ Corresponding author:mtate@gradcenter.cuny.edu \\ Published 16 December 2021; https://doi.org/10.18061/FDMC.2021.0024 \\ Author video presentation and/or other conference material: https://doi.org/10.17605/OSF.IO/75ZUS
}

\begin{abstract}
This study investigated whether adults with ADHD ("Cases") regulate emotions differently than their non-ADHD peers ("Controls"), and whether this effect is moderated by the number of years of musical training. We predicted that Cases would have poorer emotion regulation than Controls and the relation between ADHD and poor emotion regulation would be weakened with increased years of musical training. 1537 participants aged $18-65(\mathrm{M}=32, \mathrm{SD}=12$ years $)$ were recruited from the Music Universe project (www.musicaluniverse.org; Greenberg, \& Rentfrow, 2017). 57\% were female and $8 \%$ had a lifetime ADHD diagnosis. Emotion regulation was measured with the Mentalized Affectivity Scale (Greenberg et al., 2017), which assesses the ability to: identify, control and modulate, and express emotions inwardly or outwardly. More years of musical training was significantly associated with a better ability to identify emotions, irrespective of clinical status. Cases were significantly better at identifying emotions compared to Controls, but the groups did not differ in how they processed emotions. Adults with ADHD showed higher outward emotional expression, but only when number of years of music training was low/moderate. Musical training shows promise for improving how adults with ADHD express emotions, perhaps underpinned by stronger top-down mechanisms at work in emotional self- control.
\end{abstract}

KEYWORDS: ADHD, emotion regulation, musical training, self-regulation.

\section{Introduction}

Attention-deficit/ Hyperactivity Disorder (ADHD) is a common, impairing neurodevelopmental disorder characterized by symptoms of inattention, hyperactivity and impulsivity (American Psychiatric Association, 2013). ADHD affects approximately 7\% of children (Thomas et al., 2015) and 2.8\% of adults worldwide
(Fayyad et al., 2017). However, during recent years, the largest increases in ADHD prevalence have been observed in adults (not children) (Chung et al., 2019). Between 2007 and 2016, there was a 123\% increase in the prevalence of ADHD in adults (Chung et al., 2019).

In addition to the core symptoms of ADHD, 34 to $70 \%$ of adults with ADHD experience emotion regulation difficulties (Shaw et al., 2014). Emotion regulation has been defined "as an individual's ability to modify an emotional state so as to promote adaptive, goal-oriented behaviors" (Shaw et al., 2014, p. 279). The study of emotion regulation difficulties among adults with elevated ADHD behaviors is critical as emotion dysregulation is an independent predictor of their functional impairment, above and beyond inattention and hyperactivity/impulsivity (Barkley \& Fischer, 2010; O’Neill \& Rudenstine, 2019). For example, Barkley and Fischer (2010) found that among young adults with a childhood history of hyperactivity, emotional impulsiveness was an independent predictor of overall impairment, and specifically, their home life, intimate and other interpersonal relationships, financial management, driving, and engagement in community activities. Among adults seeking treatment at a community-based mental health clinic, O'Neill and Rudenstine (2019) found that emotion regulation deficits mediated the association between inattention and severity of anxiety and depression, as well as the relationship between inattention and problems with friends, family and romantic partners. Taken together, findings highlight the need for interventions to address not only the core symptoms of ADHD, but also the associated emotion regulation difficulties.

One intervention that may show some promise is music training. The music therapy and music cognition literature describe music's impact on various domains 
of cognitive functioning, including attention, language processing, working memory, as well as emotion regulation (see Moreno \& Bidelman, 2014 for a review). To date, however, no one has studied the impact of music training on emotion regulation among individuals with ADHD.

The aims of the current study were to examine if adults with a lifetime diagnosis of ADHD regulate emotions differently than their peers who have no mental health diagnosis, and if this effect is moderated by the amount years of musical training received. We hypothesized that adults with a lifetime diagnosis of ADHD ("Cases") would have poorer emotion regulation than adults with no history of mental health problems ("Controls"), and that this relation would be moderated by the number of years of music training. That is, the relation between ADHD and poor emotion regulation would be weakened as people had more years of music training.

\section{Method}

Participants

Participants are from the Musical Universe project, and self-reported measures voluntarily on www.musicaluniverse.org, from November 2015 and ending July 2016. Participants were recruited via international media outlets (e.g., CNN, IFLScience). The current sample comprises 2129 participants who ranged in age from $18-65$ years $(\mathrm{M}=32, \mathrm{SD}=12$ years) and who completed the Mentalized Affectivity Scale (Greenberg, Kolasi, Hegsted, Berkowitz, \& Jurist, 2017), the emotion regulation questionnaire utilized in the current study.

Just over half $(57 \%)$ of the sample identified as female and $43 \%$ identified as male. Nearly three quarters $(72.1 \%)$ of the participants identified as white; $1.7 \%$ identified as Black/African American; $4.4 \%$ as Latinx; $9.9 \%$ as Asian; $<1 \%$ as Native American/Pacific Islander; $11.4 \%$ identified as belonging to a racial group other than those listed. The sample was generally well educated with the majority of participants having an undergraduate degree $(35.4 \%)$ or graduate degree $(25.9 \%)$. Approximately one fifth of the sample had a High School diploma or equivalent (20.8\%); 16.5\% were currently attending high school, and $1.4 \%$ had not completed high school.

\section{Materials}

The Music Universe database comprises 17 sections that broadly measure a participant's musical preferences, personality, emotion regulation, and demographic information. ADHD diagnosis was assessed by a single question, which asked participants to indicate if they had ever been diagnosed with any of the mental health disorders listed, one of which was ADHD.

Musical training was assessed by a single self-report item. Participants stated how many years of musical training they had received in their lifetime.

Emotion Regulation was measured using the Mentalized Affectivity Scale (MAS; Greenberg et al., 2017). The 60-item self-report assessment measures three dimensions of emotion regulation, based on Jurist's (2005) theory of Mentalized Affectivity (MA): Identifying, Processing and Expressing emotions. Identifying emotions involves, at its most basic level, labeling emotions, but also includes exploring deeper emotional meaning such as, "Why am I feeling this way?" Processing Emotions measures one's ability to modulate or regulate the duration and intensity of an emotion. Expressing Emotions measures the ways in which one communicates emotions on a spectrum from outwardly to inwardly. Participants were asked to rate the extent they agreed or disagreed with each statement on a 7-point scale. High scores for both the Identifying and Processing domains represent a stronger ability to label emotions and modulate the intensity of those emotions respectively. For Expressing Emotions, lower scores reflect outward expression of emotions whereas higher scores reflect greater inward expression of emotions. The MAS was validated in a non-clinical sample of 2,000 individuals.

\section{Procedure}

Participants completed self-report measures voluntarily on the internet by going to www.musicaluniverse.org. The present study examined basic demographic information (e.g., age, gender, race/ethnicity), clinical diagnoses held by the individual, years of formal music training received, and emotion regulation capacity.

\section{Data Analysis}

Secondary analysis of the Musical Universe database was performed. Emotion regulation was parsed into three dimensions - Identifying, Processing and Expressing, each of which was normally distributed across the sample. Bivariate correlation analysis examined relations among the three MAS dimensions. An independent-samples t-test was conducted to compare each emotion regulation dimension between Cases and Controls. Years of formal music training was not normally distributed across the sample therefore a 
Mann-Whitney $U$ test examined whether there were differences in years of formal music education between Cases and Controls. Moderation analysis was conducted using Hayes' (2018) PROCESS to test whether ADHD Diagnosis (IV) was associated with differences in the three dimensions of Emotion Regulation (DV), and to see whether these relations were moderated by a continuous measure of the number of years of music training (M). Number of years of music training was mean centered and the HC3-estimator was used to account for non-normality of residuals. 10,000 bootstrap samples were taken to estimate the indirect effect.

\section{Results}

125 participants reported an ADHD diagnosis ("Cases"), and 1448 participants reports having no mental health diagnosis ("Controls"). A wide range of years of formal music training was observed from 0 years to 41 years of training. The median years of music training participants completed was $3(\mathrm{IQR}=0-7)$ but $35 \%$ of participants had 0 years of music training. A Mann-Whitney U test showed no significant differences in years of formal music education between Cases $(\mathrm{Mdn}=4.75)$ and Controls $(\mathrm{Mdn}=4.50), \mathrm{U}\left(\mathrm{N}_{\mathrm{ADHD}}=105\right.$, $\left.\mathrm{N}_{\text {Controls }}=1252\right)=69100, \mathrm{z}=.89, p=.37$.

Bivariate correlation analysis revealed significant, but weak relations among the three dimensions of emotion regulation. For Identifying and Processing emotions, $r=.24, p<.0001$; Identifying and Expressing emotions, $\quad r=-.24, \quad p<.001 ;$ and Processing and Expressing emotions, $r=-.22, p<.001$.

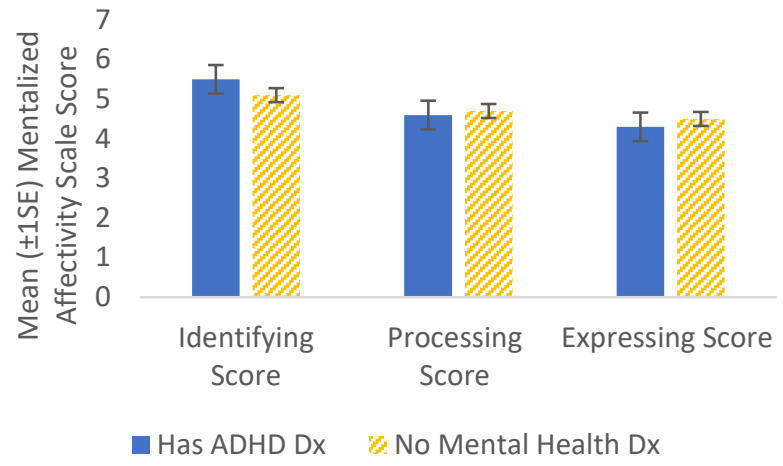

Figure 1: Mean $( \pm 1 S E)$ scores for the Mentalized Affectivity Scale domains - Identifying, Processing and Expressing - as a function of ADHD diagnosis.

Mean differences in the three domains of emotion regulation were calculated between Cases and Controls (see Figure 1). Compared to Controls, the Cases: scored higher on the Identifying emotions scale, $t(1571)=-4.66$, $p<.0001, \mathrm{~d}=.47$; showed no difference in Processing of emotions, $t(1571)=1.49, p=.14, \mathrm{~d}=.13$; and scored significantly lower on the Expressing emotions scale, $t(1571)=2.58, p=.01, \mathrm{~d}=.23$.

Moderation analyses were carried out to determine whether music training moderated the association between ADHD and emotion regulation (see Table 1).

Table 1: PROCESS Moderation Results

\begin{tabular}{|c|c|c|c|c|}
\hline $\begin{array}{l}\text { Model/ } \\
\text { Predictor }\end{array}$ & Coefficient & $t$ & $p$ & $\begin{array}{l}\text { 95\% CI } \\
\text { (Lower, } \\
\text { Upper) }\end{array}$ \\
\hline \multicolumn{5}{|l|}{ Identifying } \\
\hline Constant & 5.11 & 205.46 & $<.0001$ & $5.05,5.15$ \\
\hline $\begin{array}{l}\text { ADHD } \\
\text { diagnosis }\end{array}$ & .33 & 3.96 & .0001 & $.17, .49$ \\
\hline $\begin{array}{l}\text { Music } \\
\text { Training }\end{array}$ & .007 & 1.39 & .17 & $-.003, .02$ \\
\hline $\begin{array}{l}\text { ADHD x } \\
\text { Music }\end{array}$ & -.02 & -1.2 & .23 & $-.06, .01$ \\
\hline \multicolumn{5}{|l|}{ Processing } \\
\hline Constant & 4.17 & 214.87 & $<.0001$ & $4.67,4.77$ \\
\hline $\begin{array}{l}\text { ADHD } \\
\text { diagnosis }\end{array}$ & -0.14 & -1.52 & .13 & $-.31, .04$ \\
\hline $\begin{array}{l}\text { Music } \\
\text { Training }\end{array}$ & 0.006 & 1.49 & .14 & $-.002, .01$ \\
\hline $\begin{array}{l}\text { ADHD x } \\
\text { Music }\end{array}$ & -.02 & -.96 & .34 & $-.05, .02$ \\
\hline \multicolumn{5}{|l|}{ Expressing } \\
\hline Constant & 4.52 & 159.43 & $<.0001$ & $4.46,4.57$ \\
\hline $\begin{array}{l}\text { ADHD } \\
\text { diagnosis }\end{array}$ & -0.22 & -2.13 & .03 & $-.43,-.02$ \\
\hline $\begin{array}{l}\text { Music } \\
\text { Training }\end{array}$ & -0.006 & -1.14 & .26 & $-.02, .004$ \\
\hline $\begin{array}{l}\text { ADHD x } \\
\text { Music }\end{array}$ & 0.04 & 2.25 & .02 & $.005, .08$ \\
\hline
\end{tabular}

A significant model for Identifying emotions was seen, $F(3,1353)=6.61, p=.0002$. ADHD diagnosis was a unique predictor, such that Cases identified emotions significantly better than Controls. There was no main effect of Music Training on identifying emotions, and no significant ADHD x Music Training interaction.

A non-significant model for Processing emotions was obtained, $F(3,1353)=1.62, p=.18$ (see Table 1$)$. Neither ADHD diagnosis nor years of music training 
were associated with Processing and no significant ADHD diagnosis $x$ Music Training interaction emerged.

A significant model for Expressing emotions was obtained, $F(3,1353)=3.52, p=.015$ (see Table 1). A significant association between ADHD diagnosis and Expressing emotions was qualified by a significant ADHD diagnosis $x$ years of Music Training interaction (see Figure 2). Cases had significantly lower Expressing scores (i.e., more outward emotional expression) than Controls when years of formal music training was low $\left(16^{\text {th }}\right.$ percentile, $t(1353)=-3.18, p=.002,95 \%$ CI:-. 67 , .16.) or moderate $\left(50^{\text {th }}\right.$ percentile, $\mathrm{t}(1353)=-2.70$, $p=.007,95 \%$ CI:-.50, -.08.). When years of formal music training was high $\left(84^{\text {th }}\right.$ percentile, $t(1353)=.05$, $p=.96,95 \%$ CI: $-.29, .30)$, no differences in Expressing was seen between Cases and Controls.

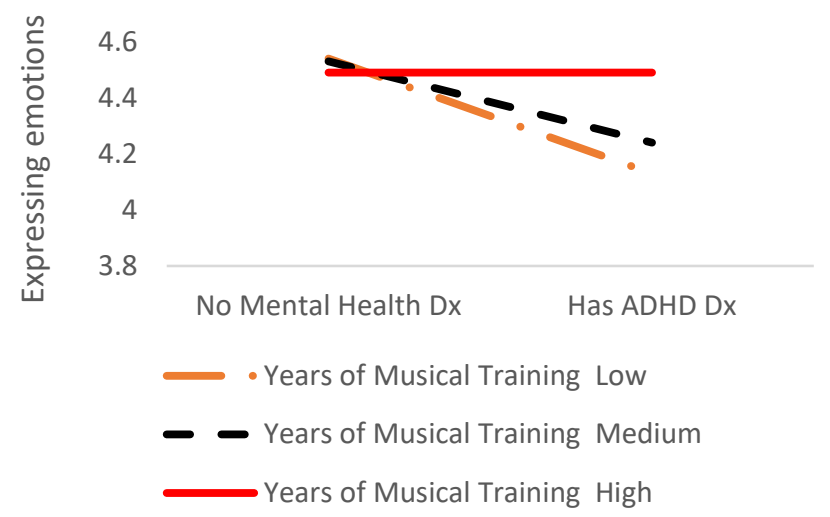

Figure 2: Interaction between ADHD diagnosis and years of music training on Expressing domain of Mentalized Affectivity Scale.

\section{Discussion}

The aim of the current study was to determine whether the association between ADHD and emotion regulation capacity is moderated by years of formal music training.

First, we found an association between ADHD diagnosis and Identifying emotions. Somewhat surprisingly, those with a lifetime history of ADHD ("Cases") were found to identify emotions significantly better than those without a mental health diagnosis ("Controls"). Music training was not associated with Identifying emotions, and nor did music training moderate the effect of ADHD on capacity to identify emotions. Greenberg and colleagues (2017) described one's ability to identify emotions as an ability to recognize emotions and make sense of emotions. It is possible that adults with a lifetime diagnosis of ADHD are attuned to their own emotion distress, but not to that of others. This is an area for future research.

The processing emotions domain measured an individual's ability to modulate and regulate emotions. This includes changing emotions in different ways, such as by duration or intensity (Greenberg et. al, 2017). Adults with a lifetime diagnosis of ADHD did not differ in how they process emotions when compared to individuals without a mental health diagnosis, which was surprising given the commonly reported emotion dysregulation among individuals with ADHD. For example, Shushakova, Ohrmann, and Pedersen (2017) found that compared to healthy controls, adults with ADHD showed more elevated emotional reactivity to negative stimuli, had decreased ability to evaluate their capacity to regulate emotions or to reappraise emotional situations, and that their use of emotion regulation strategies took greater cognitive effort.

In the present study, the lack of an effect of lifetime ADHD diagnosis on emotion Processing may be due to sample bias. It is possible that individuals who selfselected to take part in this study may have been relatively higher functioning than other adult ADHD samples.

Finally, research has shown that adults with a lifetime diagnosis of ADHD exhibited greater emotional impulsivity and emotion dysregulation, which is expressed primarily through irritability, aggression and temper outbursts (Mitchell, Robertson, Anastopolous, Nelson-Gray, \& Kollins, 2012; Shaw et al., 2014). Consistent with this literature, results of our analysis show that Cases showed greater outward emotion expression. This may reflect the inhibitory control deficits experienced by many with ADHD (Barkley, 1997).

Last, the relation between lifetime ADHD diagnosis and emotion expression was moderated by years of music training in a dose-dependent manner. When number of years of music training was high, no difference was observed between Cases and Controls, perhaps reflecting stronger top-down control of emotions after many years of training. Shaw and colleagues (2014) report that individuals with ADHD have deficits in their ability to adjust top-down regulation in response to different emotional stimuli. Research on music training has shown it can lead to improvement of top-down cognitive processes in general populations (e.g., Kim, Wigram \& Gold, 2008). Thus, it is possible that those adults with a lifetime 
diagnosis of ADHD who had many years of music training developed better self-regulation capacity.

\section{Conclusion}

To our knowledge this is one of the first studies to explore the effects of formal music training on emotion regulation among individuals with ADHD. Given the possible connection between deficits in cognitive processes, such as working memory and inhibitory control, and emotion dysregulation (Barkley, 1997; Shaw et al., 2014), future research should examine the extent to which inhibitory control mediates the effects of formal music training on emotion regulation among individuals with ADHD. Further, research should focus on whether music training affects symptom remittance thus providing evidence for changes in lifetime symptom trajectory. Also, future research should be conducted to determine if music training could serve as an efficacious intervention that improves both emotion dysregulation in individuals with ADHD as well as the core ADHD symptoms.

\section{References}

American Psychiatric Association. (2013). Diagnostic and statistical manual of mental disorders (5th ed.). https://doi.org/10.1176/appi.books.9780890425596

Barkley R. A. (1997). Behavioral inhibition, sustained attention, and executive functions: constructing a unifying theory of ADHD. Psychological bulletin, 121(1), 65-94. https://doi.org/10.1037/0033-2909.121.1.65

Barkley, R. A., \& Fischer, M. (2010). The unique contribution of emotional impulsiveness to impairment in major life activities in hyperactive children as adults. Journal of the American academy of child and adolescent psychiatry, 49(5), 503-513. https://doi.org/10.1016/j.jaac.2010.01.019

Chung, W., Jiang, S. F., Paksarian, D., Nikolaidis, A., Castellanos, F. X., Merikangas, K. R., \& Milham, M. P. (2019). Trends in the Prevalence and Incidence of Attention-Deficit/Hyperactivity Disorder Among Adults and Children of Different Racial and Ethnic Groups. JAMA network open, 2(11), e1914344. https://doi.org/10.1001/jamanetworkopen.2019.14344

Fayyad, J., Sampson, N. A., Hwang, I., Adamowski, T., Aguilar-Gaxiola, S., Al-Hamzawi, A., Andrade, L. H., Borges, G., de Girolamo, G., Florescu, S., Gureje, O., Haro, J. M., Hu, C., Karam, E. G., Lee, S., Navarro-Mateu, F., O'Neill, S., Pennell, B. E., Piazza, M., Posada-Villa, J., ... WHO World Mental Health Survey Collaborators (2017). The descriptive epidemiology of DSM-IV Adult ADHD in the World Health Organization World Mental Health Surveys. Attention deficit and hyperactivity disorders, 9(1), 47-65. https://doi.org/10.1007/s12402016-0208-3

Greenberg, D. M., Kolasi, J., Hegsted, C. P., Berkowitz, Y., \& Jurist, E. L. (2017). Mentalized affectivity: A new model and assessment of emotion regulation. PloS one, 12(10), e0185264. https://doi.org/10.1371/journal.pone.0185264

Jurist, E. L. (2005). Mentalized affectivity. Psychoanalytic psychology, 22(3), 426-444. https://doi.org/10.1037/07369735.22.3.426

Kim, J., Wigram, T., \& Gold, C. (2008). The effects of improvisational music therapy on joint attention behaviors in autistic children: a randomized controlled study. Journal of autism and developmental disorders, 38(9), 1758-1766. https://doi.org/10.1007/s10803-008-0566-6

Mitchell, J. T., Robertson, C. D., Anastopolous, A. D., Nelson-Gray, R. O., \& Kollins, S. H. (2012). Emotion dysregulation and emotional impulsivity among adults with attention- deficit/hyperactivity disorder: Results of a preliminary study. Journal of psychopathology and behavioral assessment, 34, 510-519. https://doi.org/10.1007/s10862-012-9297-2

Moreno, S., \& Bidelman, G. M. (2014). Examining neural plasticity and cognitive benefit through the unique lens of musical training. Hearing research, 308, 84-97. https://doi.org/10.1016/j.heares.2013.09.012

O’Neill, S., Rudenstine, S. (2019). Inattention, emotion dysregulation and impairment among urban, diverse adults seeking psychological treatment. Psychiatry research, 282, 112631. https://doi.org/10.1016/j.psychres.2019.112631

Shaw, P., Stringaris, A., Nigg, J., \& Leibenluft, E. (2014). Emotion dysregulation in attention deficit hyperactivity disorder. The American journal of psychiatry, 171(3), 276293. https://doi.org/10.1176/appi.ajp.2013.13070966

Shushakova, A., Ohrmann, P., \& Pedersen, A. (2018). Exploring deficient emotion regulation in adult ADHD: electrophysiological evidence. European archives of psychiatry and clinical neuroscience, 268(4), 359-371. https://doi.org/10.1007/s00406-017-0826-6

Thomas, R., Sanders, S., Doust, J., Beller, E., \& Glasziou, P. (2015). Prevalence of attention-deficit/hyperactivity disorder: a systematic review and meta-analysis. Pediatrics, 135(4), e994-e1001. 